\title{
A Mediastinal Liposarcoma Resected Using a Double Approach with a Thoracoscope
}

\author{
${ }^{1}$ Department of Laboratory Medicine, Shinshu University Hospital, \\ Matsumoto, Japan \\ 2 Department of Thoracic Surgery, Shinshu University School of \\ Medicine, Matsumoto, Japan \\ ${ }^{3}$ Department of Breast and Endocrine Surgery, Shinshu University \\ School of Medicine, Matsumoto, Japan \\ Thorac Cardiovasc Surg Rep 2013;2:46-49.
}

Shiho Asaka ${ }^{1}$ Kazuo Yoshida ${ }^{2}$ Mashiro Hashizume ${ }^{2}$ Ken-ichi Ito ${ }^{3}$

\begin{abstract}
Keywords

- mediastinal tumor

- sarcoma

- thoracoscopy/VATS

We report a case of a 45-year-old woman who underwent a complete resection of a liposarcoma using thoracoscopic and cervical approaches. General checkup and computed tomography revealed a large mediastinal tumor occupying the thoracic outlet, which had reached the posterior thyroid region, and another small tumor at a subcarina. A cervical method for evaluating a neck lesion and a thoracoscopic (videoassisted thoracic surgery) approach for assessing a mediastinal lesion were performed. This double approach provided excellent visualization and enabled us to perform fine manipulation even within the narrow thoracic outlet region. The patient was disease free at 11 months after surgery.
\end{abstract}

\begin{abstract}
Address for correspondence Kazuo Yoshida, MD, PhD, Department of Thoracic Surgery, Shinshu University School of Medicine, 3-1-1 Asahi Matsumoto 3908621, Japan (e-mail: kxy13@shinshu-u.ac.jp).
\end{abstract}

\section{Introduction}

Liposarcomas rarely arise in the mediastinum and comprise less than $1 \%$ of all mediastinal tumors. ${ }^{1,2}$ Complete surgical resection is thought to be the best treatment for liposarcoma because the role of adjuvant therapy has not been established for these tumors. Due to the tendency for liposarcomas to be large in size, a long incision is usually required for complete resection of these tumors. We here present a case of a mediastinal liposarcoma, which was located from the neck to the mid mediastinum, and which was resected completely using a double thoracoscopic (video-assisted thoracic surgery [VATS]) and cervical approach.

A 45-year-old woman presented at a community hospital with a mediastinal mass detected during a general checkup. She had no prior symptoms. A chest X-ray revealed a large mass shadow in her right chest. Chest computed tomography (CT) imaging showed a heterogeneous, regular-shaped mediastinal tumor (lesion A), occupying the thoracic outlet, and which had attained a posterior thyroid region location. An additional tumor was also detected by $\mathrm{CT}$ at a subcarinal region (lesion B) ( - Fig. 1A), considered to be a lymph node. Magnetic resonance imaging (MRI) showed lesion A as isointense on T1W1, heterogeneous high intensity on T2W1, and lesion $B$ as high intensity on T2W1, which revealed no notable

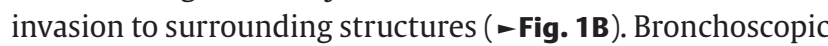
and transcervical fine needle aspiration cytology and biopsy did not detect malignancy. These findings indicated that the lesion was a soft tissue tumor, either a solitary fibrous tumor or a sarcoma of unknown origin.

The cervical approach via collar incision was initially performed before VATS approach. Because the mediastinal part of lesion A was larger than its cervical part, we concluded that the tumor should be pulled out from neck into the thorax. The tumor showed no adhesion or invasion to the tissue surrounding the cervical region and hence could be divided easily ( - Fig. 1C). After dividing the tumor, the collar incision was closed and a lateral position was adopted for the VATS procedure. Three VATS ports were placed in the middle portion of the right chest ( - Fig. 1D). In the thoracic cavity, the thoracic outlet was occupied by the tumor, which was almost free from the surrounding organs, except for fibrous adhesion received

July 2, 2013

accepted

August 19, 2013

published online

October 25, 2013
DOI http://dx.doi.org/ 10.1055/s-0033-1358605. ISSN 2194-7635.

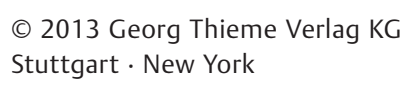

License terms

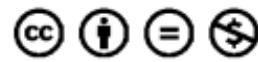



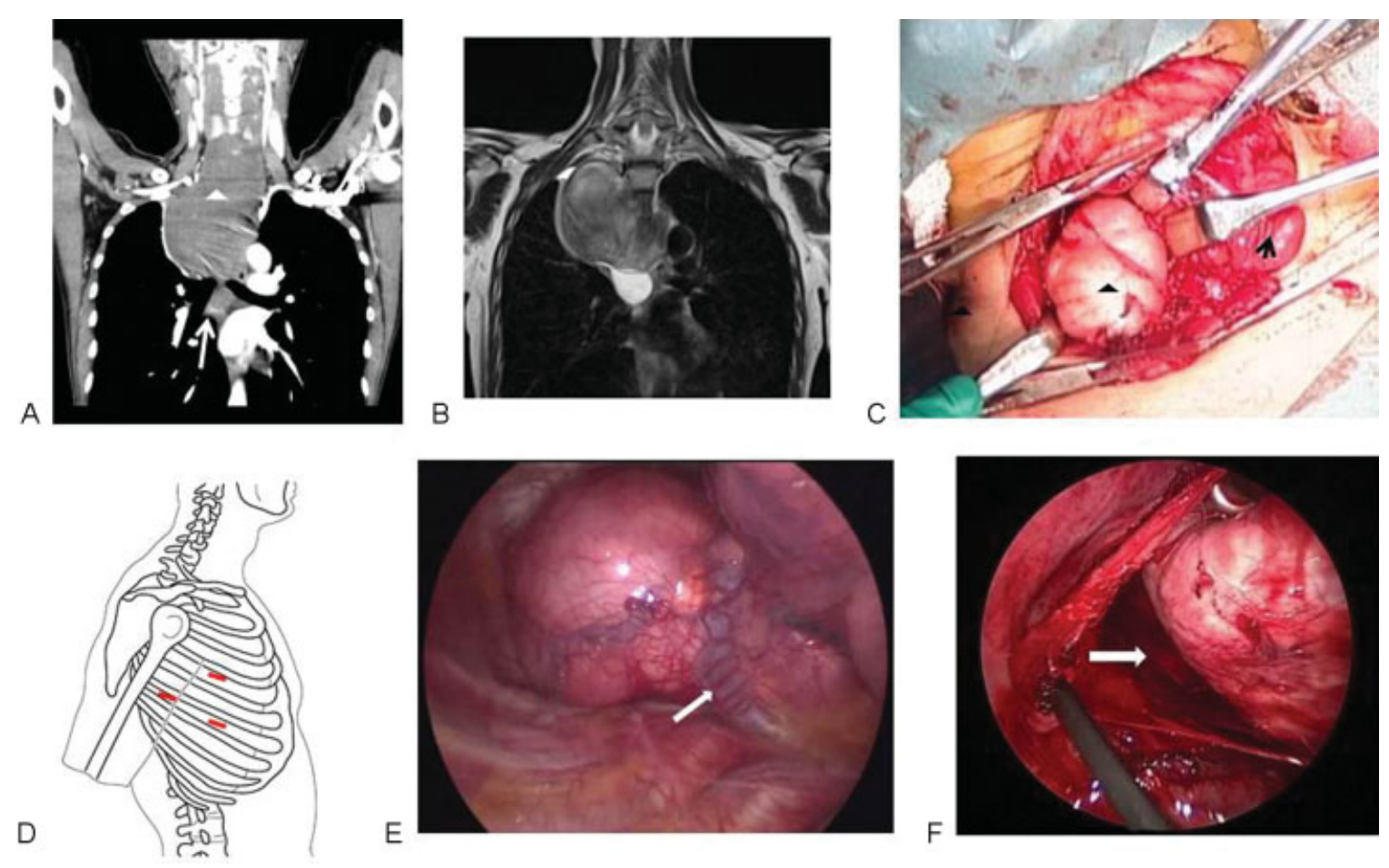

Fig. 1 (A) Coronal view of a chest computed tomography of the patient revealed a large mediastinal tumor occupying the thoracic outlet, which attained a posterior thyroid level at the cervical region (lesion A; arrowhead). Another smaller tumor was located at a subcarinal region (lesion B; arrow). (B) Coronal view of a chest magnetic resonance imaging finding. T2W1 revealed heterogeneous high intensity for lesion A, and notable high intensity for lesion B, which did not reveal distinct invasion to surrounding structures. (C) Intraoperative view of the dissection of the cervical region. The tumor (arrow head) and thyroid (arrow) are indicated. (D) Three ports (red line) were placed in the middle portion of the right chest for video-assisted thoracic surgery (VATS), two at the seventh intercostal space (anterior and posterior axillary line each) and one at the fifth intercostal space (middle axillary line). (E) Intraoperative VATS view of the dissection of the mediastinum, fine view of the area around the thoracic apex without a blind spot, and thoracic apertures. The arrow indicates the azygos vein. (F) The arrow indicates "the tunnel" from the thoracic outlet to the neck.

in places (-Fig. 1E). The dissection was preceded along the parietal pleura until the apical thorax. At the apical thorax, we carefully tunneled through the thoracic outlet to the neck (-Fig. 1F). Lesion A was then pulled out gently into the thorax. Lesion B which was next to, but independent of, lesion A was also extirpated. Both tumors were completely resected with negative margin thoracoscopically.

Macroscopically, lesion A, measuring $12.7 \times 10 \mathrm{~cm}$, was encapsulated and the cut surface was solid and uniformly white (-Fig. 2A). Lesion B, measuring $3.5 \times 2.7 \mathrm{~cm}$, appeared pale, yellow, and gelatinous with a spotty hemorrhage (-Fig. 2E). Histology revealed, lesion A was a fibrous tumor which consisted of spindle cells with mild nuclear atypia (-Fig. 2B), and lesion B was an adipocytic tumor having abundant myxoid stroma and scattered hyperchromatic stromal cells and atypical lipoblasts (-Fig. 2F). Immunohistochemistry (IHC) with CDK4 and MDM2 antibodies showed weak nuclear positivity in lesion A (-Fig. 2C, D) and B (-Fig. 2G, H). Additionally, fluorescence in situ hybridization (FISH) revealed co-amplification of MDM2 and CDK4 in both lesions (-Fig. 2C, D, G, H). Finally, she was pathologically diagnosed with dedifferentiated liposarcoma forming two separate lesions.

The postoperative course for this patient was uneventful with no serious wound pain. She was discharged from the hospital on postoperative day 11 , and was disease free at 11 months postsurgery.

\section{Discussion}

A mediastinal liposarcoma accounts for only approximately $9 \%$ of primary sarcomas of the mediastinum. ${ }^{1,2}$ Recent advances in the molecular genetic characterization have led to the classification of liposarcoma into three main histogenic subtypes: well-differentiated/dedifferentiated liposarcoma, myxoid liposarcoma, and pleomorphic liposarcoma. ${ }^{3}$ The prognosis is highly dependent on histological subtypeWell-differentiated/dedifferentiated liposarcoma has a particularly protracted clinical course, and myxoid and pleomorphic subtypes tend to progress rapidly. ${ }^{2,4}$ Therefore, adequate pathological classification is essential for determining prognosis and therapy. In our current case, with their histology, it is considered that lesion B was well-differentiated liposarcoma, and lesion A was a low-grade dedifferentiated counterpart of lesion B. Demonstration of MDM2-CDK4 by IHC and FISH confirmed the diagnosis.

As with most soft tissue tumors, since radio and/or chemotherapy are believed to be ineffective, complete surgical excision is the appropriate treatment for liposarcoma. Although adjuvant therapy was not undertaken in our patient 


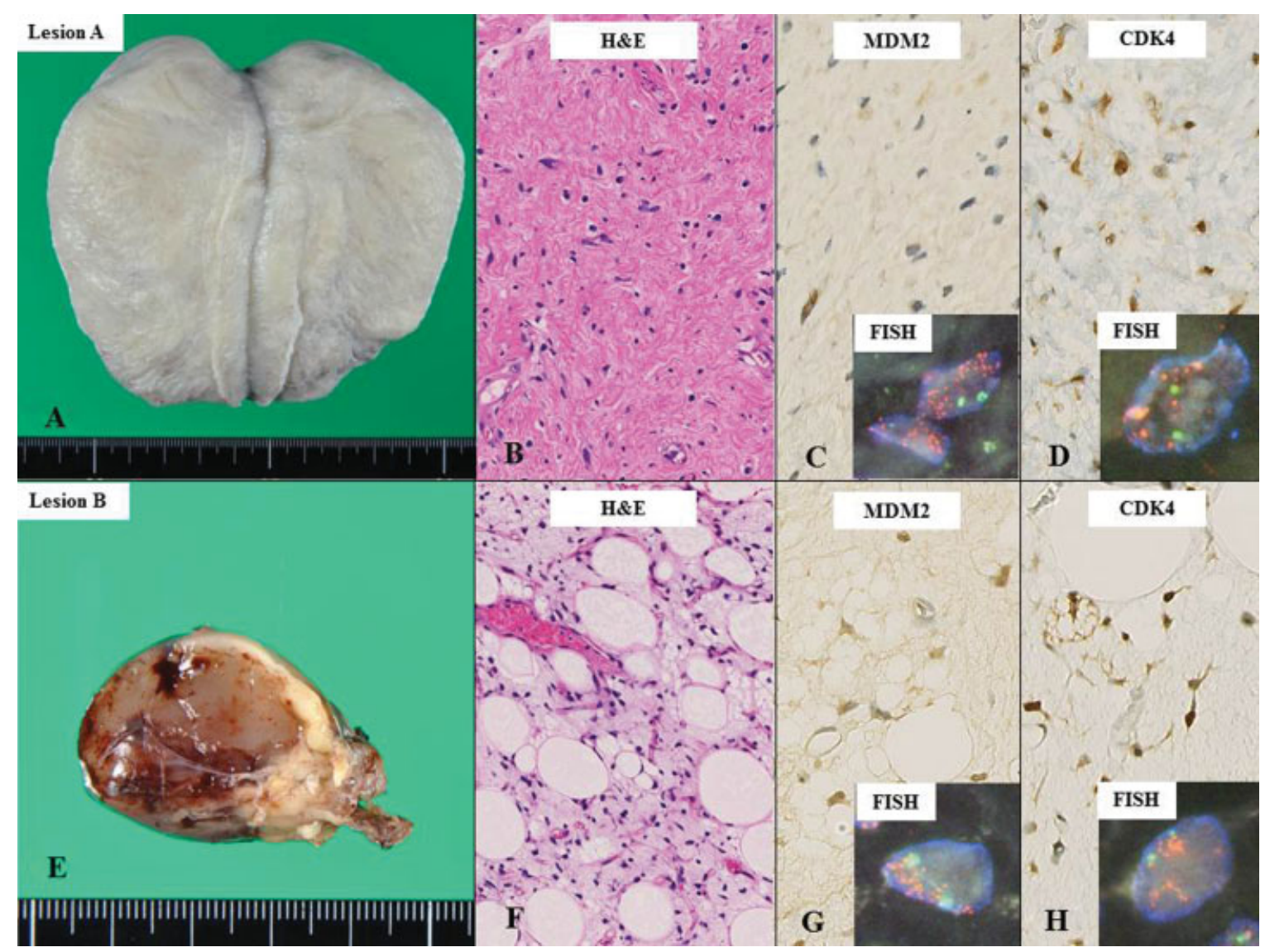

Fig. 2 Macroscopical findings: (A) Gross picture, histology, immunohistochemistry (IHC), and fluorescence in situ hybridization (FISH) with MDM2 and CDK4 probes of lesion A. (B) In lesion A, spindle-shaped cells with mild nuclear atypia are arranged with fibrillary collagenous stroma, and there is no adipocytic component. (C, D) Tumor cells of lesion A show focal and weak positivity for MDM2 and CDK4. (E) Gross picture, histology, IHC, and FISH with MDM2 and CDK4 probes of lesion B. (F) Lesion B is composed of adipocytes in a variety of sizes, scattered hyperchromatic stromal cells, and atypical lipoblasts with abundant myxoid matrix; mimicking well-differentiated liposarcoma or myxoid liposarcoma. (G, H) Tumor cells of lesion B show diffuse and weak positivity for MDM2 and CDK4. In FISH, the orange signals represent MDM2 or CDK4, and the green signals represent centromeres of chromosome 12. Both lesions show amplification of MDM2 (C, G) and CDK4 (D, H).

because the tumor was resected completely with negative margin, a careful follow-up plan was definitely warranted.

Liposarcomas tend to be large in size, and a large thoracotomy is, therefore, often required for a complete excision with a negative margin. For these reasons, anterior or posterior thoracotomy, sternotomy, or clamshell procedures are usually considered. ${ }^{5}$ However, these approaches can result in postoperative pain, and morbidity and a prolonged hospital stay because of rib spreading and a long incision. Decker et al reported a successful resection for a large liposarcoma located in an inferior thorax by thoracoscopic intervention. ${ }^{2}$ These authors reported that a minimally invasive thoracoscopic procedure resulted in less pain and a shorter hospital stay. In our present patient, the classical neck collar incision and lateral thoracotomy would have been considered in most settings due to the large size, location, and suspicious adhesion of the lesion. Even with these approaches, however, visibility would be expected to be poor and involve a blind spot, especially at the thoracic apex, because of the large size and immovability of the tumor. However, the VATS approach generated excellent visualization without a blind spot and enabled us to perform a safe and more precise operation, even in a narrow space.

In conclusion, the VATS approach should be considered in cases with a complete and less invasive resection of a large liposarcoma located at a thoracic apical region.

\section{Acknowledgment}

We thank Akishi Ooi, Department of Molecular and Cellular Pathology, Kanazawa University Graduate School of Medical Science, Ishikawa, Japan for technical support of fluorescence in situ hybridization.

\section{References}

1 Burt M, Ihde JK, Hajdu SI, et al. Primary sarcomas of the mediastinum: results of therapy. J Thorac Cardiovasc Surg 1998;115(3): 671-680

2 Decker JR, de Hoyos AL, Decamp MM. Successful thoracoscopic resection of a large mediastinal liposarcoma. Ann Thorac Surg 2011;92(4):1499-1501 
3 Weiss SW. Enzinger and Weiss's Soft Tissue Tumors. Philadelphia, PA: Mosby/Elsevier; 2008

4 Boland JM, Colby TV, Folpe AL. Liposarcomas of the mediastinum and thorax: a clinicopathologic and molecular cytogenetic study of 24 cases, emphasizing unusual and diverse histologic features. Am J Surg Pathol 2012;36(9):1395-1403
5 Ohta Y, Murata T, Tamura M, Sato H, Kurumaya H, Katayanagi K. Surgical resection of recurrent bilateral mediastinal liposarcoma through the clamshell approach. Ann Thorac Surg 2004;77(5): 1837-1839 